\title{
Critical properties in long-range hopping Hamiltonians
}

\author{
E. Cuevas* \\ Departamento de Física, Universidad de Murcia, E-30071 Murcia, Spain.
}

Received 15 March 2004, revised 26 April 2004, accepted 26 April 2004

Published online 25 June 2004

PACS 64.60.Ak, 71.30.+h, 72.15.Rn

Some properties of $d$-dimensional disordered models with long-range random hopping amplitudes are investigated numerically at criticality. We concentrate on the correlation dimension $d_{2}$ (for $d=2$ ) and the nearest level spacing distribution $P_{c}(s)$ (for $d=3$ ) in both the weak $\left(b^{d} \gg 1\right)$ and the strong $\left(b^{d} \ll 1\right)$ coupling regime, where the parameter $b^{-d}$ plays the role of the coupling constant of the model. It is found that (i) the extrapolated values of $d_{2}$ are of the form $d_{2}=c_{d} b^{d}$ in the strong coupling limit and $d_{2}=d-a_{d} / b^{d}$ in the case of weak coupling, and (ii) $P_{c}(s)$ has the asymptotic form $P_{c}(s) \sim \exp \left(-A_{d} s^{\alpha}\right)$ for $s \gg 1$, with the critical exponent $\alpha=2-a_{d} / b^{d}$ for $b^{d} \gg 1$ and $\alpha=1+c_{d} b^{d}$ for $b^{d} \ll 1$. In these cases the numerical coefficients $A_{d}, a_{d}$ and $c_{d}$ depend only on the dimensionality.

Copyright line will be provided by the publisher

\section{Introduction}

Quantum phase transitions in disordered electronic systems remain one of the central problems in condensed-matter physics. Considerable attention has recently focused on both the energy levels and the critical eigenfunctions, which strongly fluctuate near the critical point and thus have multifractal scaling properties [1-7]. Wave-function statistics can be characterized through the set of generalized fractal dimensions $d_{q}$ which are associated with the scaling of the $q$-th moment of the wave-function intensity. A complete knowledge of $d_{q}$ is equivalent to a complete physical characterization of the fractal [1].

Among all the fractal dimensions, the correlation dimension $d_{2}$ of the eigenfunctions plays the most prominent role, since it has been related to the level compressibility [8-11], the spatial dispersion of the diffusion coefficient [12] and the anomalous spreading of a wave-packet at the mobility edge [13]. Multifractality is also related to the probability overlap of eigenstates with energy separation much greater than the average separation level. In particular, $d_{2}$ describes these density correlations. It has been shown that for multifractal eigenstates these correlations decay slowly no matter how sparse those states are [14].

Short-range correlations in the energy levels, which are closely related to the localization properties of the corresponding wave-functions [15-17], can be described by the critical distribution $P_{c}(s)$ of the normalized spacings $s$. The large $s$ asymptotic behavior of this distribution is still an open question and the subject of discussion [18].

Metal-insulator transitions (MIT's) depend on the dimensionality and symmetries of the system and can occur in both the strong disorder and the weak disorder regime (strong-coupling or weak-coupling regime, respectively, of the corresponding field-theoretical description) as well as in the intermediate regime. Each regime is characterized by its respective coupling strength [19].

The disorder-induced MIT is usually investigated for Hamiltonians with short-range, off-diagonal matrix elements (e.g., the canonical Anderson model). Other Hamiltonians exhibiting an MIT in arbitrary dimension $d$ are those that include long-range hopping terms [20-25]. The effect of long-range hopping

* Corresponding author: e-mail: ecr@um.es 
on localization was originally considered by Anderson [22] for randomly distributed impurities in $d$ dimensions with the $V\left(\boldsymbol{r}-\boldsymbol{r}^{\prime}\right) \sim\left|\boldsymbol{r}-\boldsymbol{r}^{\prime}\right|^{-\beta}$ hopping interaction. It is known [22,23,25] that all states are extended for $\beta<d$, whereas for $\beta>d$ the states are localized. Thus, the MIT can be studied by varying the exponent $\beta$ at fixed disorder strength. At the transition line $\beta=d$, a real-space renormalization group can be constructed for the distribution of couplings [23,24]. These models are the most convenient for studying critical properties numerically since the exact quantum critical point is known $(\beta=d)$ and, in addition, they allow the low-dimensional cases to be treated, thus using larger system sizes and reducing the numerical effort.

Although a great progress in understanding critical properties of the $1 d$ long-range random hopping Hamiltonian has recently been made $[5,7,9,11,26-37]$ explicit results for the $2 d$ and $3 d$ systems are still lacking. Our aim was to investigate the two previously mentioned important quantities, the correlation dimension and nearest level spacing distribution, at the MIT, which have been left unexplored. The last quantity could help us to solve the existing controversy about the form of $P_{c}(s)$ at the MIT.

The paper begins by first giving the model used for the calculations in Sec. 2 The results for the correlation dimension and for the critical level spacing distribution are presented in Sec. 3 and 4 respectively. Finally, Sec. 5 summarizes our findings.

\section{The model}

In order to fully represent the mesoscopic systems we introduce an explicit dependence on dimensionality $d$ in the widely studied power-law random banded matrix (PRBM) ensemble $[5,7,9,11,26-36]$ (for closely related models see also Ref. [37]). Thus, we consider a generalization to $d$ dimensions of this ensemble. The corresponding Hamiltonian, which describes non-interacting electrons on a disordered $d$-dimensional square lattice with random long-range hopping, is represented by real symmetric matrices, whose entries are randomly drawn from a normal distribution with zero mean, $\left\langle\mathcal{H}_{i j}\right\rangle=0$, and a variance which depends on the distance between the lattice sites $\boldsymbol{r}_{i}$

$$
\left\langle\left|\mathcal{H}_{i j}\right|^{2}\right\rangle=\frac{1}{1+\left(\left|\boldsymbol{r}_{i}-\boldsymbol{r}_{j}\right| / b\right)^{2 \beta}}\left\{\begin{array}{ll}
\frac{1}{2}, & i \neq j \\
1, & i=j
\end{array} .\right.
$$

Using field theoretical methods [5, 6,9-11,23, 25, 26], the PRBM model was shown to undergo a sharp transition at $\beta=d$ from localized states for $\beta>d$ to delocalized states for $\beta<d$. This transition shows all the key features of the Anderson MIT, such as multifractality of the eigenfunctions and non-trivial spectral compressibility at criticality. In what follows, we focus on the critical value $\beta=d$. The parameter $b^{d}$ in Eq. (1) is an effective bandwidth that serves as a continuous control parameter over a whole line of criticality, i.e, for an exponent equal to $d$ in the hopping elements $\mathcal{H}_{i j} \sim b^{d}$ [23]. Furthermore, it determines the critical dimensionless conductance in the same way as the dimensionality labels the different Anderson transitions. Each regime is characterized by its respective coupling strength, which depends on the ratio $\left(\left\langle\left|\mathcal{H}_{i i}\right|^{2}\right\rangle /\left\langle\left|\mathcal{H}_{i j}\right|^{2}\right\rangle\right)^{1 / 2} \propto b^{-d}$ between diagonal disorder and the off-diagonal transition matrix elements of the Hamiltonian [19].

Many real systems of interest can be described by Hamiltonians (1). Among such systems are optical phonons in disordered dielectric materials coupled by electric dipole forces [38], excitations in two-level systems in glasses interacting via elastic strain [39], magnetic impurities in metals coupled by an $r^{-3}$ Ruderman-Kittel-Kasuya-Yodida interaction [40], and impurity quasiparticle states in two-dimensional disordered $d$-wave superconductors [41]. It also describes a particle moving fast through a lattice of Coulomb scatterers with power-law singularity [25], the dynamics of two interacting particles in a $1 d$ random potential [42] and a quantum chaotic billiard with a non-analytic boundary [43].

The two limiting cases of the $1 d$ model, $b \gg 1$ and $b \ll 1$, which correspond to the weak and the strong disorder limits, respectively, can be studied via the mapping onto the supermatrix $\sigma$-model for $b$ 
large $[5,26]$ and using the renormalization-group method of Refs. [23, 24] for small $b$. In particular, one finds the following result for the correlation dimension $d_{2}$ at the center of the band

$$
d_{2}=\left\{\begin{array}{ll}
1-\frac{1}{\pi b}, & b \gg 1 \\
2 b, & b \ll 1
\end{array} .\right.
$$

Thus, for the $1 d$ version of Hamiltonian (1), which possesses the line of critical points $0<b<\infty, d_{2}$ changes from 0 to the system dimensionality $d=1$ as $b$ increases. Eq. (2) has been numerically confirmed by several groups using exact diagonalization $[6,27,29,36]$. We stress that, unlike the $1 d$ PRBM model, it has not until now been possible to analytically solve the $2 d$ and $3 d$ disordered models with long-range transfer terms.

The system sizes used are $L=24,36,48,72$ and 96 in $2 d$, and $L=8,12,14$ and 16 in $3 d$, whereas $b^{d}$ ranges in the interval $0.01 \leq b^{d} \leq 10$. We consider a small energy window, containing about $10 \%$ of the states around the center of the spectral band. The number of random realizations is such that the number of critical levels and eigenstates included for each $L$ is roughly $1.3 \times 10^{6}$, except for the larger system size in both dimensions, for which this number is about $6 \times 10^{5}$. In order to reduce edge effects, periodic boundary conditions are included. The power-law nature of Hamiltonians (1) did not allow us to use efficient algorithms, such as the Lanzos algorithm, which is usually applied to the study of the MIT in the Anderson model, due to the large degree of sparcity of the corresponding Hamiltonian. Instead, we use standard diagonalization subroutines.

\section{The correlation dimension}

In this section we investigate critical fluctuations in the eigenfunctions of the two-dimensional model (1) in terms of multifractal measures, focusing on the correlation dimension $d_{2}$. At the MIT, where the natural length scale (the localization length) diverges, strong fluctuations in the wave-functions $\psi_{\mu}(\boldsymbol{r})$ appear on all length scales. These fluctuations can be characterized by a set of inverse participation ratios (IPR) [44]

$$
I_{\mu}(q)=\int_{\Omega} d^{2} r\left|\psi_{\mu}(\boldsymbol{r})\right|^{2 q} \propto L^{-\tau_{q}}, \quad \tau_{q}=d_{q}(q-1),
$$

where $d_{q}$ is a set of generalized fractal dimensions. The index $\mu$ labels different eigenfunctions and $\Omega$ denotes a 2-dimensional region with linear dimension $L$. Equation (3) is valid for individual states and for their ensemble average since the spectrum of multifractal dimensions has universal features for states in the vicinity of the MIT [3]. The inverse of $I_{\mu}(2)$ roughly equals the number of nonzero wave-function components, for which reason it is a widely accepted measure of the extension of the states. Note that in a good metal, for which eigenfunctions are ergodic, the IPR scale with size $L$ as $I_{\mu}(q) \propto L^{-d(q-1)}$, whereas in an insulator, with localized states, $I_{\mu}(q) \propto L^{0}$.

For the computation of $\tau_{q}$ we used the standard box-counting procedure [3], first dividing the system of $L^{2}$ sites into $N_{l}=(L / l)^{2}$ boxes of linear size $l$ and determining the box probability of the wave function in the $i$ box by $p_{i}(l)=\sum_{\boldsymbol{r}}\left|\psi_{\mu}(\boldsymbol{r})\right|^{2}$, where the summation is restricted to sites within that box, and $\psi_{\mu}(\boldsymbol{r})$ denotes the amplitude of an eigenstate with energy $\epsilon_{\mu}$ at site $\boldsymbol{r}$. The normalized $q$-th moments of this probability constitute a measure. From this, the mass exponents $\tau_{q}(L)$, which encode generalized dimensions $d_{q}(L)=\tau_{q}(L) /(q-1)$, can be obtained [45]

$$
\tau_{q}(L)=\lim _{\delta \rightarrow 0} \frac{\ln \sum_{i=1}^{N_{l}} p_{i}^{q}(l)}{\ln \delta},
$$




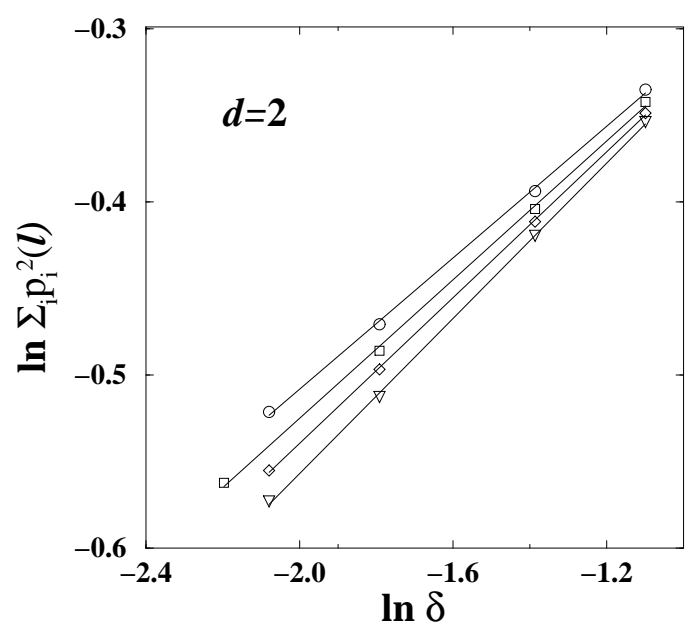

Fig. $1 \ln \delta$ dependence of $\ln \sum_{i=1}^{N_{l}} p_{i}^{2}(l)$ in the strongcoupling regime $\left(b^{2}=0.05\right)$ for different system sizes: $L=24$ (circles), 36 (squares), 48 (diamonds) and 96 (triangles). The straight lines whose slopes correspond to the values of $d_{2}(L)$ are linear fits to Eq. (4).

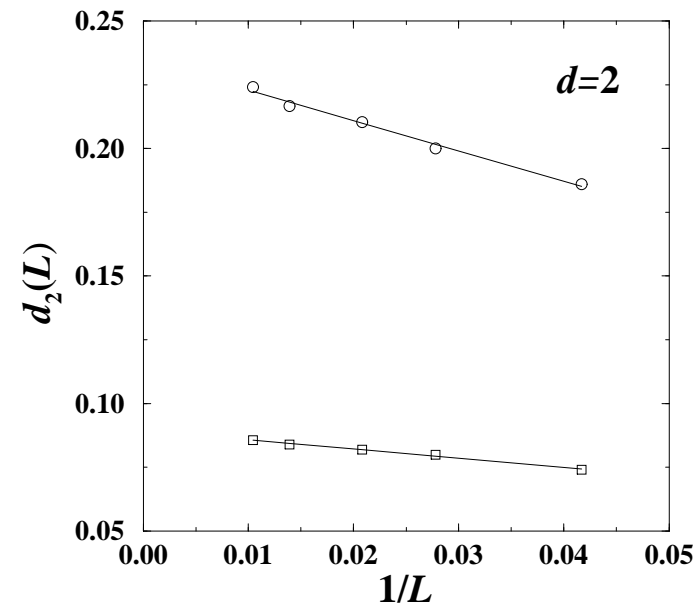

Fig. 2 Finite-size corrections to the correlation dimension $d_{2}(L)$ in the strong-coupling regime $b^{2}=0.02$ (squares) and $b^{2}=0.05$ (circles); solid lines are fits to Eq. (6).

where $\delta=l / L$ denotes the ratio of the box sizes and the system size. It should be made clear that the calculation of $\tau_{q}(L)$ is suitable only if the conditions [3]

$$
a \ll l<L \ll \xi,
$$

are satisfied, where $\xi$ is the localization or correlation length and $a$ is the lattice spacing (or any microscopic length scale of the system). In practice, $\tau_{q}(L)$ is found by performing a linear regression of $\ln \sum_{i=1}^{N_{l}} p_{i}^{q}(l)$ with $\ln \delta$ in a finite interval of $\delta$. In order to properly satisfy the previous conditions (4) and (5), we take $\delta$ to be in the interval $(0.1,0.4)$. Since we are mainly interested in the correlation dimension, we shall restrict ourselves to the value $q=2$ and so $d_{2}=\tau_{2}$.

Using the exact eigenstates of Hamiltonian (1) obtained from numerical diagonalizations, we evaluate, for each value of $b$ and $L$, the numerator on the right-hand side of Eq. (4) for decreasing box sizes, and then calculate $d_{2}(L)$ from the slope of the graph of the numerator vs $\ln \delta$. Figure 1 provides an example of the $\ln \delta$ dependence of $\ln \sum_{i=1}^{N_{l}} p_{i}^{2}(l)$ in the strong-coupling regime $\left(b^{2}=0.05\right)$ for different system sizes: $L=24$ (circles), 36 (squares), 48 (diamonds) and 96 (triangles). Clearly, there is no ambiguity in the determination of the slopes that correspond to the values of $d_{2}(L)$. These slopes are summarized in Fig. 2.

In Ref. [36] it was shown that the finite-size corrections to $\tau_{2}(L)$ are of power-law type $\left(\sim L^{-1}\right)$ for the $1 d$ PRBM model. A similar decay was found for the multifractal spectrum $f(\alpha)$ and its singularity strength $\alpha$ in $d=1$ and 2 [35]. Thus, in order to predict the asymptotic values of $d_{2}$, a curve of the form

$$
d_{2}(L)=d_{2}+a_{2} / L
$$

is proposed. Other forms, such as exponential $\left(\sim e^{-a_{2} L}\right)$ or inverse logarithmic $\left(\sim 1 / \ln a_{2} L\right)$ have been rejected since we have checked that none of them can adequately describe the $d_{2}$ size behavior.

In Fig. 2, we represent the finite-size corrections for the correlation dimension $d_{2}(L)$ of the $2 d$ disordered system described by Eq. (1) in the strong-coupling regime $b^{2}=0.02$ (squares) and 0.05 (circles). 


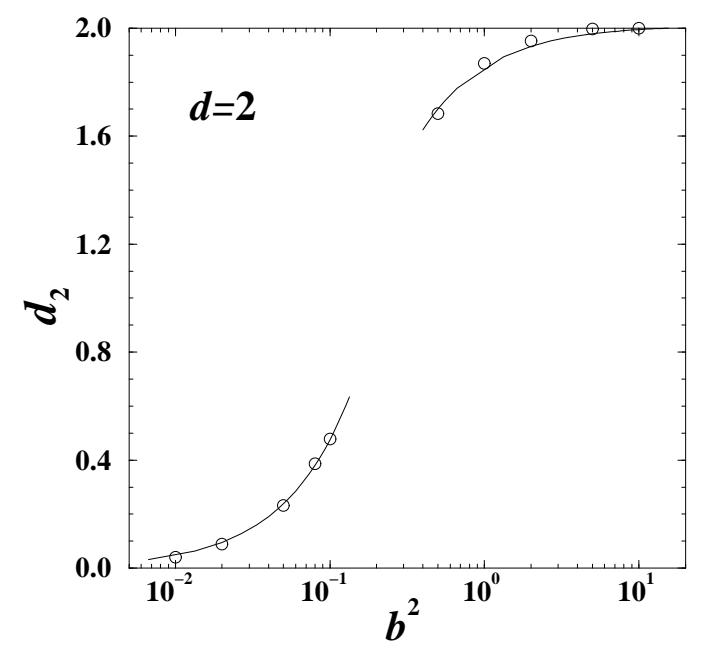

Fig. $3 b^{2}$ dependence of the correlation dimension $d_{2}$ (circles) for the $2 d$ disordered model. Solid lines are fits to Eq. (7) corresponding to the limiting cases of weak $\left(b^{2} \gg 1\right)$ and strong $\left(b^{2} \ll 1\right)$ disorder.

Note that significant finite-size effects are present. The straight lines are linear fits to Eq. (6) and intercept the vertical axis at $0.0894 \pm 0.0004$ and $0.235 \pm 0.002$, respectively.

The extrapolated values of $d_{2}$, as obtained from the previous fits, are shown in Fig. 3 (circles) as a function of the disorder parameter $b^{2}$ of the PRBM model. They clearly change continuously from 0 as $b^{2} \rightarrow 0$ to the system dimensionality $d=2$ as $b^{2} \rightarrow \infty$. In the two limiting cases of weak $\left(b^{2} \gg 1\right)$ and strong $\left(b^{2} \ll 1\right)$ disorder regimes $d_{2}$ can be well fitted by

$$
d_{2}=\left\{\begin{array}{ll}
2-\frac{a_{2}}{b^{2}}, & b^{2} \gg 1 \\
c_{2} b^{2}, & b^{2} \ll 1
\end{array},\right.
$$

respectively. These fits are shown as solid lines in Fig. 3 The fitting parameters are $a_{2}=0.16 \pm 0.06$ and $c_{2}=4.76 \pm 0.07$. Note the similarity of Eqs. (7) with the corresponding to the $1 d$ case, Eqs. (2).

\section{The level spacing distribution}

This section is devoted to the analysis of short-range energy level correlations in the three-dimensional model. The emphasis is on the large $s$ asymptotic behavior of the level spacing distribution at the mobility edge. We first review existing analytical results concerning this distribution.

On the localized side of the transition, states with close energy levels are typically localized at different parts of space and have an exponentially small overlap. Their levels are therefore uncorrelated and the corresponding spacings are distributed according to the Poisson law

$$
P_{\mathrm{P}}(s)=\exp (-s) .
$$

In contrast, in the metallic regime, the large overlap of delocalized states, which are essentially structureless, induces correlations in the spectrum, leading to the well known level repulsion effect. If the system is invariant under rotation and under time-reversal symmetry (orthogonal symmetry), the normalized spacings $s$ follow Wigner-Dyson statistics at the infinite system size limit

$$
P_{\mathrm{W}}(s)=\frac{\pi}{2} s \exp \left(-\frac{\pi}{4} s^{2}\right) .
$$

At the disorder-induced MIT, the statistics of energy levels changes drastically and presents distinct features reflecting criticality of the theory. This statistics is characterized by a third universal (i.e., independent 
of the system size and of the details of the Hamiltonian model) distribution $P_{c}(s)$, which is different from both Wigner-Dyson statistics and the Poisson statistics $[16,46]$. On the one hand, the influence of the MIT on the spectral properties was studied in Refs. $[16,46]$ by means of the impurity diagram technique combined with scaling assumptions. In these studies, it was conjectured that

$$
P_{c}(s) \sim \exp (-\kappa s), \quad s \gg 1
$$

with $\kappa \approx 3.3$, the reason for such behavior being that the Thouless energy at the transition point is of the order of the average level spacing $\left(\epsilon_{c} / \Delta \approx 1\right)$, and so the levels's repulsion is effective only for $s \lesssim 1$.

A short-range plasma model with interaction only between closest neighbors [47-50] suggest that the universality connected with the spectral fluctuations at the MIT is the intermediate spectral statistics $P_{c}(s)=4 s \exp (-2 s)$.

On the other hand, by mapping the energy level distribution onto the Gibbs distribution for a classical one-dimensional gas with a repulsive pairwise interaction, Ref. [51] derived the following asymptotic form for $P_{c}(s)$

$$
P_{c}(s) \sim \exp \left(-A_{d} s^{\alpha}\right), \quad s \gg 1,
$$

where the coefficient $A_{d}$ depends only on the dimensionality $d$, and where the critical exponent $\alpha$, which ranges in the interval $1<\alpha<2$, is related to the correlation length exponent $\nu$ and to the dimensionality through $\alpha=1+(d \nu)^{-1}$.

Neither as regards the numerical description of $P_{c}(s)$ is there any consensus. The exponential decay, Eq. 10], of $P_{c}(s)$ has been confirmed by most groups at different MIT's (see Refs. [52-56] and references therein), while an exponent $\alpha \approx 1.2$ has been found in Refs. [57,58] from a fit in the whole range of spacings to a distribution of the form $P_{c}(s)=B s \exp \left(-A s^{\alpha}\right)$ or, indirectly, from the two-point correlation function of the density of states [59]. Anyway, the behavior (11) with some nontrivial $1 \leq \alpha \leq 2$ is what one would expect at the mobility edge.

It should be pointed out that MIT's generically take place at strong disorder (conventional Anderson transition, quantum Hall transition, transition in $d=2$ for electrons with strong spin-orbit coupling, etc.). In this regime, the predicted [51] exponent $\alpha=1+(d \nu)^{-1}$ slightly deviates from unity, making it relatively difficult to see on the numerically calculated tails of $P_{c}(s)$ (e.g, at the standard Anderson transition in $3 d$ $\alpha \approx 1.2$ ). To overcome this problem, it is necessary to investigate transitions which occur at the opposite limit (weak coupling regime). This area has recently been investigated for the model (1) when $d=1$ and 2 [18], and an exponent $\alpha$ close to the Wigner-Dyson value $\alpha=2$ has been found at large values of $b$ and $b^{2}$, respectively.

Here, we make a similar study for the more realistic and interesting case $d=3$. From results of detailed high precision numerical investigations, we will show unambiguously that Eq. (11) is indeed correct while the validity of Eq. (10) is limited to the case of very strong disorder (strictly at the limit of infinity coupling strength). In addition, we find that the exponent $\alpha$ in Eq. (11) continuously varies between 1 and 2 as the coupling strength of the Hamiltonian model changes from 0 to $\infty$.

For the computation of $P_{c}(s)$, we unfold the spectrum in each case to a constant density, and rescale it so as to have the mean spacing equal to unity. In order to diminish the magnitude of the relative fluctuations and to analyze the asymptotic behavior in detail, it is more convenient to consider the cumulative level spacing distribution function $I(s)=\int_{s}^{\infty} P\left(s^{\prime}\right) d s^{\prime}$. Note that the integration does not change the asymptotic behavior of $P(s)$. The Wigner surmise, Eq. (9), and the Poisson distribution, Eq. [8, yield $I_{\mathrm{W}}(s)=\exp \left(-\pi s^{2} / 4\right)$ and $I_{\mathrm{P}}(s)=\exp (-s)$, respectively.

Figure 4 displays our results for the integrated probability $I_{c}(s)$ of the $3 d$ model for $L=14$ at $b^{3}=$ 0.02, 0.05 and 5, which are depicted consecutively from bottom to top. Dotted and dashed lines, which correspond to $I_{\mathrm{W}}(s)$ and $I_{\mathrm{P}}(s)$, respectively, are given for comparison. A gradual crossover in the large $s$ tail of $I_{\mathrm{c}}(s)$ from the Poisson to the Wigner-Dyson limiting forms as one increases the inverse coupling constant $b^{3}$ of the model can clearly be seen. So, we can therefore expect an exponent $\alpha$ in Eq. (11), 


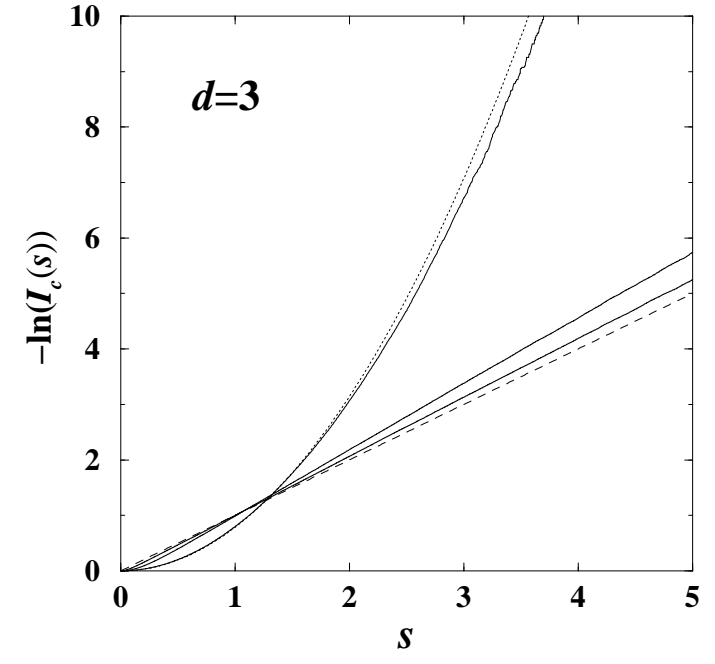

Fig. 4 Integrated probability $I_{c}(s)$ of the $3 d$ system for $L=14$ at $b^{3}=0.02,0.05$ and 5 (from bottom to top). Dotted and dashed lines are $I_{\mathrm{W}}(s)$ and $I_{\mathrm{P}}(s)$, respectively.

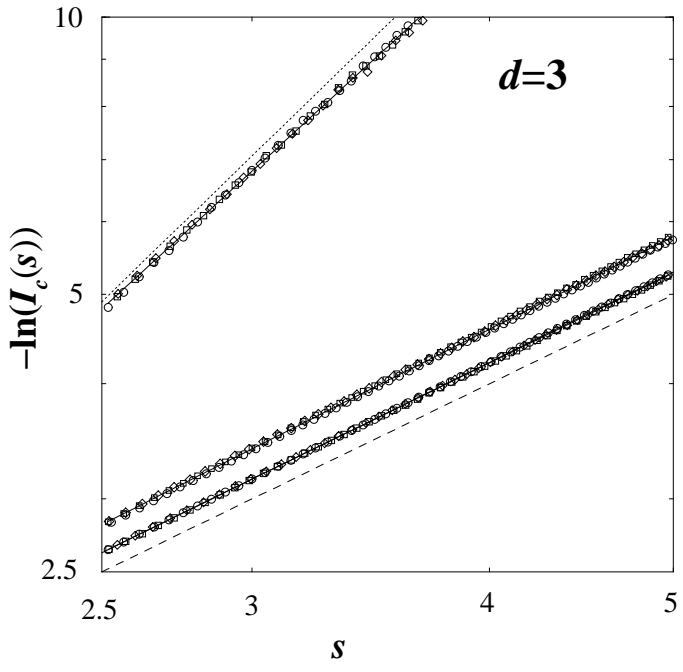

Fig. 5 Log-log plot of the integrated probability $I_{c}(s)$ of the $3 d$ system at $b^{3}=0.02,0.05$ and 5 (from bottom to top) and different system sizes $L=8$ (circles), 12 (squares) and 16 (diamonds). Dotted and dashed lines are $I_{\mathrm{W}}(s)$ and $I_{\mathrm{P}}(s)$, respectively, and the straight lines are fits to Eq. [11.

which spans the interval $[1,2]$, in agreement with Ref. [51]. For the $1 d$ and $2 d$ cases the behavior is quite similar [18].

Next we consider the behavior of $I_{c}(s)$ with system size $L$. The results for $s$ large of the critical $I_{c}(s)$ for the $3 d$ model at different values of $b^{3}$ are shown in a log-log scale in Fig. 5 for different system sizes: $L=8$ (circles), 12 (squares) and 16 (diamonds). Note that $I_{c}(s)$ is an $L$-independent universal scale-invariant function that interpolates, as previously mentioned, between Wigner and Poisson limits. This result confirms the existence of a critical distribution exactly at the transition. Dotted and dashed lines correspond to $I_{\mathrm{W}}(s)$ and $I_{\mathrm{P}}(s)$, respectively. We checked that the normalized variances of $P_{c}(s)$ are indeed scale-invariant at each critical point studied $[60,61]$. The straight line behavior of the data in such a plot at all values of $b^{3}$ considered is undoubtedly consistent with a $b^{3}$ dependent exponent $\alpha$ in Eq. (11). The values of $b^{3}$ reported are $0.02,0.05$ and 5 , from bottom to top. The best fit to Eq. 111) in the interval $2.5 \lesssim s \lesssim 5$ for small $b^{3}$ and $2.5 \lesssim s \lesssim 4$ for large $b^{3}$, yields $\alpha=1.008,1.039$ and 1.901 , respectively, thus confirming the result of [51]. Note that for the large energy ranges considered, where $I_{c}(s)$ vary by one to three orders of magnitude, the quality of the fits, which are represented as solid straight lines, is evident.

Finally, the disorder dependence of the critical exponent $\alpha$, as obtained from the previous fits for the $3 d$ system (circles) is shown in Fig. 6 in the broad range of the parameter $b^{3}$ of the $3 d$ PRBM model. It clearly changes continuously from the Poisson value $\alpha=1$ as $b^{3} \rightarrow 0$ to the Wigner-Dyson value $\alpha=2$ as $b^{3} \rightarrow \infty$. In the two limiting cases of weak $\left(b^{3} \gg 1\right)$ and strong $\left(b^{3} \ll 1\right)$ disorder regimes it can be fitted by

$$
\alpha=\left\{\begin{array}{ll}
2-\frac{a_{3}}{b^{3}}, & b^{3} \gg 1 \\
1+c_{3} b^{3}, & b^{3} \ll 1
\end{array},\right.
$$

respectively. These fits are shown as solid lines in Fig. 6 The fitting parameters are $a_{3}=0.18 \pm 0.04$ and $c_{3}=0.96 \pm 0.07$. Note that at $b^{3} \gg 1$ the condition $\left|\boldsymbol{r}_{i}-\boldsymbol{r}_{j}\right| / b \gg 1$ is not completely fulfilled for 


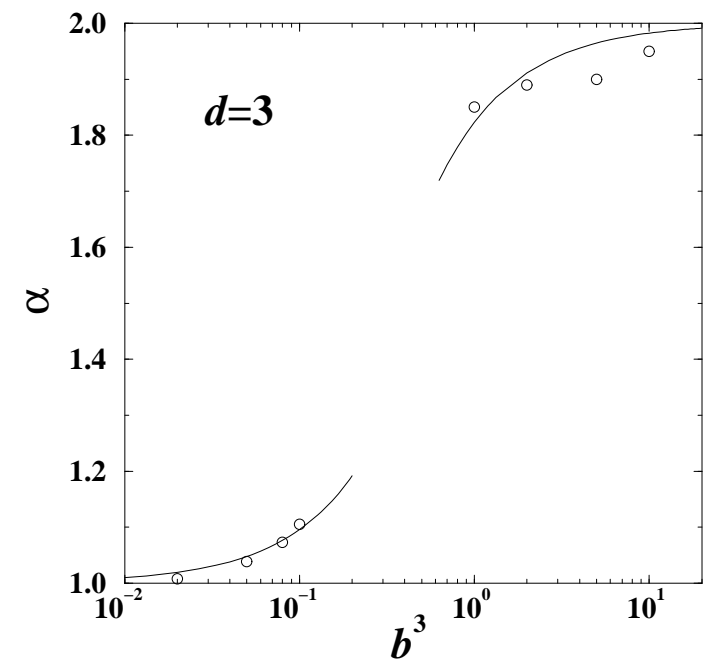

Fig. $6 b^{3}$ dependence of the critical exponent $\alpha$ (circles) for the $3 d$ disordered system. Solid lines are fits to Eq. 12. corresponding to the limiting cases of weak $\left(b^{3} \gg\right.$ $1)$ and strong $\left(b^{3} \ll 1\right)$ disorder.

the system sizes considered and for the largest $b^{3}$ reported the corresponding $\alpha$ saturate at smaller values than predicted by the previous equation. From eq. (12), the Poissonian tail of $P_{c}(s)$, eq. (10), is recovered for large spacings at the limit of very strong coupling $b^{3} \rightarrow 0$. So, we conclude that in the case of very strongly coupled Hamiltonians only, eq. 11 loses its validity and eq. (10) applies.

\section{Summary}

We have calculated the correlation dimension $d_{2}$ of the eigenfunctions and the nearest level spacing distribution $P_{c}(s)$ of non-interacting electrons on $d$-dimensional disordered models $(d=2$ and 3$)$ with longrange random transfer terms at criticality in the whole range of the coupling constant $b^{-d}$. The leading finite-size corrections to $d_{2}$ decay algebraically with exponents equal to -1 . At the infinite-size limit, it is found that $d_{2}$ is of the form $d_{2}=c_{d} b^{d}$ for small $b^{d}$ and $d_{2}=d-a_{d} / b^{d}$ for large $b^{d} . P_{c}(s)$ is found to be scale-independent at all values of $b^{-d}$. The large $s$ part of $P_{c}(s)$ obtained is shown to have an $\exp \left(-A_{d} s^{\alpha}\right)$ decay with $1 \leq \alpha \leq 2$. Finally, we determined the disorder dependence of $\alpha$ in both the strong $\left(b^{d} \ll 1\right)$ and the weak $\left(b^{d} \gg 1\right)$ coupling regimes. At the limit of very strong disorder $b^{d} \rightarrow 0$, we found that $\alpha \rightarrow 1$ and so we obtain the expected results of the Poissonian decay predicted in Refs. [16, 46].

Acknowledgements The author thanks the Spanish DGESIC for financial support through project numbers BFM200303800 and FIS2004-03117.

\section{References}

[1] H. G. E. Hentschel and I. Procaccia, Physica D 8, 435 (1983).

[2] T. C. Hasley, M. H. Jensen, L. P. Kadanoff, I. Procaccia and B. Shraiman, Phys. Rev. A 33, 1141 (1986).

[3] M. Janssen, Int. J. Mod. Phys. B 8, 943 (1994); B. Huckestein, Rev. Mod. Phys. 67, 357 (1995).

[4] V. I. Falko and K. B. Efetov, Europhys. Lett. 32, 627 (1995); Phys. Rev. B 52, 17413 (1995).

[5] A. D. Mirlin, Phys. Rep. 326, 259 (1999).

[6] F. Evers and A. D. Mirlin, Phys. Rev. Lett. 84, 3690 (2000).

[7] E. Cuevas, M. Ortuño, V. Gasparian, and A. Pérez-Garrido, Phys. Rev. Lett. 88, 016401 (2002).

[8] J. T. Chalker, V. E. Kravtsov and I. V. Lerner, Pis'ma Zh. Eksp. Teor. Fiz. 64, 355 (1996) [JETP Lett. 64, 386 (1996)].

[9] V. E. Kravtsov and K. A. Muttalib, Phys. Rev. Lett. 79, 1913 (1997).

[10] V. E. Kravtsov, Ann. Phys. (Leipzig) 8, 621 (1999). 
[11] V. E. Kravtsov and A. M. Tsvelik, Phys. Rev. B 62, 9888 (2000).

[12] J. T. Chalker and G. J. Daniell, Phys. Rev. Lett. 61, 593 (1988); J. T. Chalker, Physica A 167, 253 (1990).

[13] B. Huckestein and L. Schweitzer, Phys. Rev. Lett. 72, 713 (1994).

[14] Y. V. Fyodorov and A. D. Mirlin, Phys. Rev. B 55, R16001 (1997).

[15] B. L. Altshuler and B. I. Shklovskii, Zh. Eksp. Teor. Fiz. 91, 220 (1986) [Sov. Phys. JETP 64, 127 (1986)].

[16] B. I. Shklovskii, B. Shapiro, B. R. Sears, P. Lambrianides, and H. B. Shore, Phys. Rev. B 47, 11487 (1993).

[17] V. E. Kravtsov, I. V. Lerner, B. L. Altshuler and A. G. Aronov, Phys. Rev. Lett. 72, 888 (1994).

[18] E. Cuevas, cond-mat/0310774

[19] K. B. Efetov, Adv. Phys. 32, 53 (1983).

[20] D. A. Parshin and H. R. Schober, Phys. Rev. B 57, 10232 (1998).

[21] H. Potempa and L. Schweitzer, Phys. Rev. B 65, 201105(R) (2002).

[22] P. W. Anderson, Phys. Rev. 109, 1492 (1958).

[23] L. S. Levitov, Europhys. Lett. 9, 83 (1989); Phys. Rev. Lett. 64, 547 (1990).

[24] L. S. Levitov, Ann. Phys. (Leipzig) 8, 697 (1999).

[25] B. L. Altshuler and L. S. Levitov, Phys. Rep. 288, 487 (1997).

[26] A. D. Mirlin, Y. V. Fyodorov, F. M. Dittes, J. Quezada and T. H. Seligman, Phys. Rev. E 54, 3221 (1996).

[27] A. D. Mirlin and F. Evers, Phys. Rev. B 62, 7920 (2000).

[28] I. Varga and D. Braun, Phys. Rev. B 61, R11859 (2000).

[29] I. Varga, Phys. Rev. B 66, 094201 (2002).

[30] E. Cuevas, V. Gasparian and M. Ortuño, Phys. Rev. Lett. 87, 056601 (2001).

[31] E. Cuevas, Phys. Rev. B 66, 233103 (2002).

[32] O. Yevtushenko and V. E. Kravtsov, J. Phys. A 36, 8265 (2003).

[33] O. Yevtushenko and V. E. Kravtsov, Phys. Rev. E 69, 026104 (2004).

[34] A. M. García-García, cond-mat/0306321 (2003).

[35] E. Cuevas, Phys. Rev. B 68, 024206 (2003).

[36] E. Cuevas, Phys. Rev. B 68, 184206 (2003).

[37] J.-L. Pichard and B. Shapiro, J. Phys. I France 4, 623 (1994); M. Moshe, H. Neuberger, and B. Shapiro, Phys. Rev. Lett. 73, 1497 (1994).

[38] C. C. Yu, Phys. Rev. Lett. 63, 1160 (1989).

[39] R. N. Bhatt and P. A. Lee, Phys. Rev. Lett. 48, 344 (1982).

[40] P. Cizeau and J. P. Bouchaud, J. Phys. A 26, L187 (1993).

[41] A. V. Balatsky and M. I. Salkola, Phys. Rev. Lett. 76, 2386 (1996).

[42] I. V. Ponomarev and P. G. Silvestrov, Phys. Rev. B 56, 3742 (1997).

[43] G. Casati and T. Prosen, Physica D 131, 293 (1999). F. Borgonovi, P. Conti, D. Rebuzzi, B. Hu and B. Li, Physica D 131, 317 (1999).

[44] F. Wegner, Z. Phys. B 36, 209 (1980); C. Castellani and L. Peliti, J. Phys. A 19, L429 (1986); B. L. Altshuler, V. E. Kravtsov, and I. V. Lerner, Zh. Eksp. Teor. Fiz. 91, 2276 (1986) [Sov. Phys. JETP 64, 1352 (1986)].

[45] A. Chhabra and R. V. Jensen, Phys. Rev. Lett. 62, 1327 (1989).

[46] B. L. Altshuler, I. Kh. Zharekeshev, S. A. Kotochigova, and B. I. Shklovskii, Zh. Eksp. Teor. Fiz. 94, 343 (1988) [Sov. Phys. JETP 67, 625 (1988)].

[47] B. Grémaud and S. R. Jain, J. Phys. A 31, L637 (1998).

[48] S. R. Jain and A. Khare, Phys. Lett. A 262, 35 (1999).

[49] E. B. Bogomolny, U. Gerland and C. Schmit, Phys. Rev. E 59, R1315 (1999).

[50] G. Auberson, S. R. Jain and A. Khare, J. Phys. A 34, 695 (2001).

[51] A. G. Aronov, V. E. Kravtsov, and I. V. Lerner, Pis'ma Zh. Eksp. Teor. Fiz. 59, 39 (1994) [JETP Lett. 59, 40 (1994)]; Phys. Rev. Lett. 74, 1174 (1995). V. E. Kravtsov and I. V. Lerner, J. Phys. A, 28, 3623 (1995).

[52] S. N. Evangelou, Phys. Rev. Lett. 75, 2550 (1995).

[53] E. Hofstetter, Phys. Rev. B 54, 4552 (1996).

[54] T. Kawarabayashi, T. Ohtsuki, K. Slevin and Y. Ono, Phys. Rev. Lett. 77, 3593 (1996).

[55] I. Kh. Zharekeshev and B. Kramer, Phys. Rev. Lett. 79, 717 (1997).

[56] S. M. Nishigaki, Phys. Rev. E 59, 2853 (1999).

[57] S. N. Evangelou, Phys. Rev. B 49, 16805 (1994).

[58] I. Varga, E. Hofstetter, M. Schreiber and J. Pipek, Phys. Rev. B 52, 7783 (1995).

[59] D. Braun and G. Montambaux, Phys. Rev. B 52, 13903 (1995).

[60] E. Cuevas, Phys. Rev. Lett. 83, 140 (1999).

[61] E. Cuevas, E. Louis, and J. A. Vergés, Phys. Rev. Lett. 77, 1970 (1996). 